\title{
The $\mathrm{O}-\mathrm{Na}$ and $\mathrm{Mg}-\mathrm{Al}$ anticorrelations in turn-off and early subgiants in globular clusters ${ }^{\star}$
}

\author{
R. G. Gratton ${ }^{1}$, P. Bonifacio ${ }^{2}$, A. Bragaglia ${ }^{3}$, E. Carretta ${ }^{1}$, V. Castellani ${ }^{4}$, M. Centurion ${ }^{2}$, A. Chieffi ${ }^{5}$, \\ R. Claudi ${ }^{1}$, G. Clementini ${ }^{3}$, F. D'Antona ${ }^{6}$, S. Desidera ${ }^{1,7}$, P. François ${ }^{8}$, F. Grundahl ${ }^{9}$, S. Lucatello ${ }^{1,7}$, \\ P. Molaro ${ }^{2}$, L. Pasquini ${ }^{8}$, C. Sneden ${ }^{10}$, F. Spite ${ }^{11}$, and O. Straniero ${ }^{12}$
}

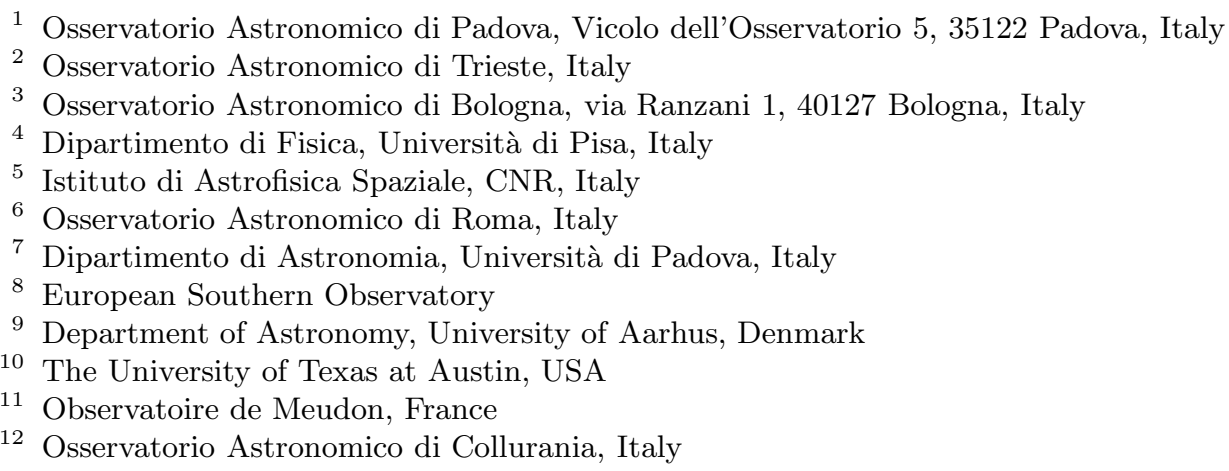

Received 18 December 2000 / Accepted 26 January 2001

\begin{abstract}
High dispersion spectra $(R \gtrsim 40000)$ for a quite large number of stars at the main sequence turn-off and at the base of the giant branch in NGC 6397 and NGC 6752 were obtained with the UVES on Kueyen (VLT $\mathrm{UT2}$ ). The $[\mathrm{Fe} / \mathrm{H}]$ values we found are $-2.03 \pm 0.02 \pm 0.04$ and $-1.42 \pm 0.02 \pm 0.04$ for NGC 6397 and NGC 6752 respectively, where the first error bars refer to internal and the second ones to systematic errors (within the abundance scale defined by our analysis of 25 subdwarfs with good Hipparcos parallaxes). In both clusters the $[\mathrm{Fe} / \mathrm{H}]$ 's obtained for TO-stars agree perfectly (within a few percent) with that obtained for stars at the base of the RGB. The $[\mathrm{O} / \mathrm{Fe}]=0.21 \pm 0.05$ value we obtain for NGC 6397 is quite low, but it agrees with previous results obtained for giants in this cluster. Moreover, the star-to-star scatter in both $\mathrm{O}$ and $\mathrm{Fe}$ is very small, indicating that this small mass cluster is chemically very homogenous. On the other hand, our results show clearly and for the first time that the $\mathrm{O}-\mathrm{Na}$ anticorrelation (up to now seen only for stars on the red giant branches of globular clusters) is present among unevolved stars in the globular cluster NGC 6752, a more massive cluster than NGC 6397. A similar anticorrelation is present also for $\mathrm{Mg}$ and $\mathrm{Al}$, and $\mathrm{C}$ and $\mathrm{N}$. It is very difficult to explain the observed $\mathrm{Na}-\mathrm{O}$, and $\mathrm{Mg}-\mathrm{Al}$ anticorrelation in NGC 6752 stars by a deep mixing scenario; we think it requires some non internal mechanism.
\end{abstract}

Key words. stars: abundances - stars: evolution - stars: population II - Galaxy: globular clusters

\section{Introduction}

Globular clusters (GCs) are (with a few significant exceptions, most notably the case of $\omega$ Cen) generally very homogenous as far as Fe-peak elements are considered; on the other hand, abundances of the lighter elements (from $\mathrm{C}$ to $\mathrm{Al}$ ) show a complex, not yet adequately explained

\footnotetext{
Send offprint requests to: R. G. Gratton, e-mail: gratton@pd.astro.it

* Based on data collected at the European Southern Observatory, Chile.
}

pattern. Rather wide variations in the strength of the $\mathrm{CH}$ and $\mathrm{CN}$ bands among stars on the red giant branch (RGB) were already noticed in the seventies (Osborn 1971, and many other references; for reviews see Smith 1987; Kraft 1994), and found to be correlated with other cluster properties (Norris 1987). More recently, a CN-CH anticorrelation was found in Main Sequence (MS) stars of NGC 6752 (Suntzeff \& Smith 1991), 47 Tuc (Cannon et al. 1998), and M 71 (Cohen 1999a) from low dispersion spectra. Even more striking is the $\mathrm{Na}-\mathrm{O}$ anticorrelation among GC red 

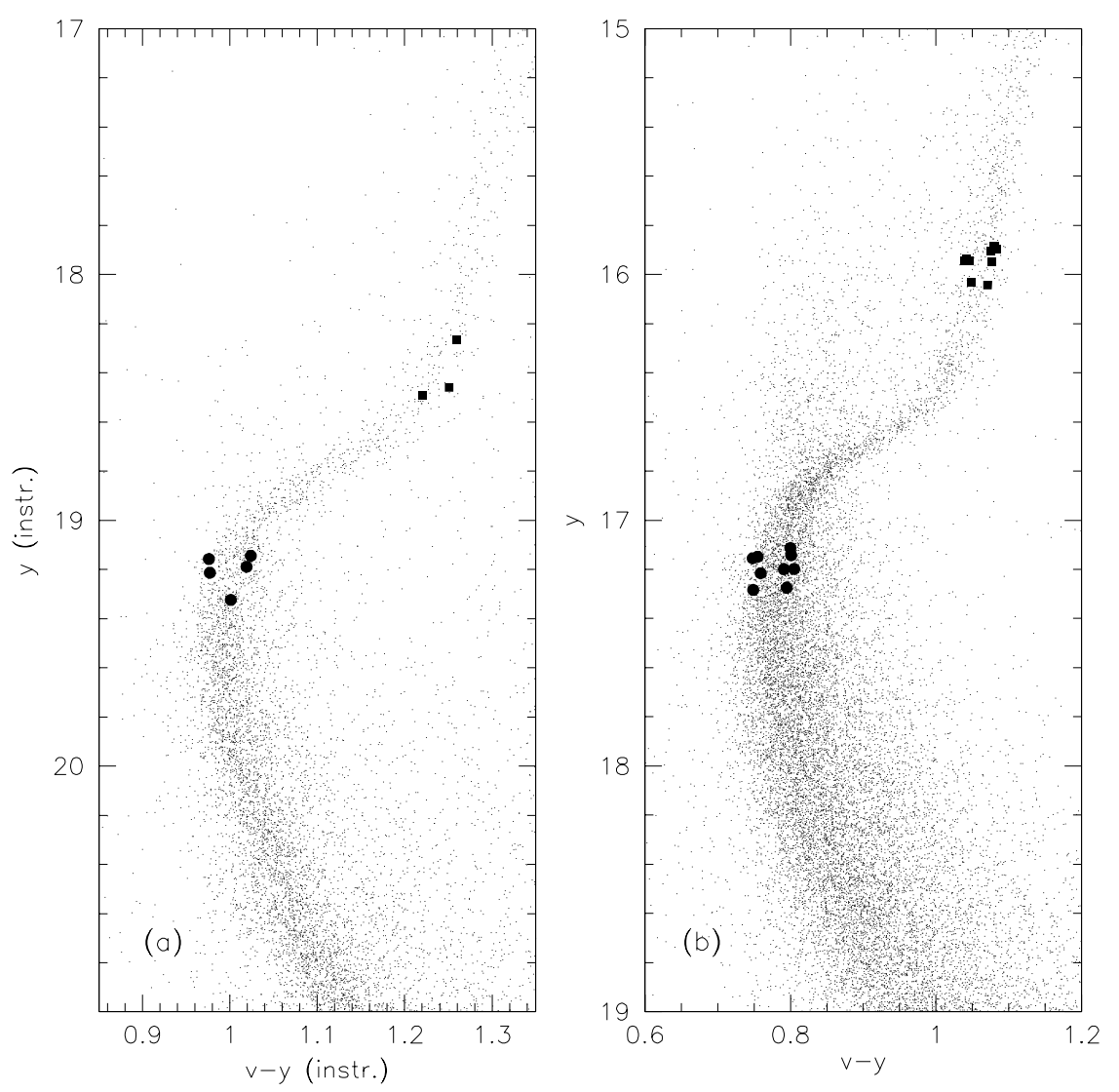

Fig. 1. Positions of program stars (large symbols) on the Strömgren colour-magnitude diagram of NGC 6397 (panel a)) and NGC 6752 (panel b)). Photometry is from Grundahl et al. (1999). Note that photometry for NGC 6397 has not been calibrated yet, so that only instrumental magnitudes are given

giants discovered by the Lick-Texas group (see e.g. Kraft et al. 1997).

These features are indications of the presence of elements processed through the complete $\mathrm{CNO}$-cycle in the atmospheres of some globular cluster stars; at temperatures where complete CNO cycle occurs, quite large amounts of Na may be produced by proton capture on ${ }^{22}$ Ne nuclei (Denissenkov \& Denissenkova 1990; Langer \& Hoffman 1995; Cavallo et al. 1996). Whatever the cause of these abundance anomalies, it appears to be related to the dense environment of GCs. In fact, field halo stars present surface abundances of $\mathrm{Li}, \mathrm{C}, \mathrm{N}, \mathrm{O}, \mathrm{Na}$ that are well explained by standard models, once a second mixing episode (only affecting $\mathrm{Li}, \mathrm{C}$ and $\mathrm{N}$ abundances) after the RGB-bump is allowed (Gratton et al. 2000). Note that the RGB bump plays an important rôle, because there the molecular weight barrier created by the deepest inward penetration of the outer convective envelope (at the base of the RGB) is canceled by the outward shift of the $\mathrm{H}$-burning shell. The absence of this barrier in the subsequent evolution allows deep mixing caused by e.g. meridional circulations (Sweigart \& Mengel 1979; Charbonnel 1994; Denissenkov \& Tout 2000). Hence, both the O-Na anticorrelation observed among giants, and the $\mathrm{C}$ and $\mathrm{N}$ anomalies observed in stars in different evolutionary phases, are peculiar to GCs.
The proposed explanations of the abundance anomalies in globular cluster stars have been oscillating for years between an "in situ" mechanism (very deep mixing in RGB stars, possibly activated by a core rotation that might be larger in cluster than in field stars) and an external source of material (either primordial proto-cluster gas or processed material from a polluting companion). Since MS stars do not have a convective envelope able to enhance $\mathrm{N}$ while depleting $\mathrm{C}$, these anomalies should be attributed mainly to a primordial origin and/or to pollution, while evolutionary mixing could possibly be responsible only for likely minor effects in RGB stars. However, rather surprisingly, an analysis of stars below the Turnoff (TO) of M 13 (Cohen 1999b) shows no variations in the strength of $\mathrm{CN}$ and $\mathrm{CH}$ molecular features, likely excluding primordial variation of $\mathrm{C}$ and $\mathrm{N}$ abundance in this cluster, which instead presents the clearest $\mathrm{Na}-\mathrm{O}$ anticorrelation on the RGB (Kraft et al. 1997), and a range in the strength of $\mathrm{CN}$ and $\mathrm{CH}$ bands among giants (Suntzeff 1981). While the result of Cohen is very surprising, the published analysis is only qualitative. Very recently, Dr. J. Cohen kindly provided us with a copy of her new quantitative analysis of these spectra in advance of publication: this analysis shows that large variations in the abundances of $\mathrm{C}$ and $\mathrm{N}$ may indeed be accomodated within the observed upper limit for star-to-star variations 
in the $\mathrm{CN}$ and $\mathrm{CH}$ bands in $\mathrm{M} 13$ main-sequence stars. However, in general, the relation between the $\mathrm{Na}-\mathrm{O}$ and $\mathrm{C}$ $\mathrm{N}$ anticorrelation is not yet well established. Observation of a $\mathrm{Na}-\mathrm{O}$ anticorrelation among main sequence stars of globular clusters would be crucial, clearly ruling out the deep mixing scenario.

Earlier explorative studies of stars close to the TO and at the base of the giant branch of a few clusters have been presented by King et al. (1998), Deliyannis et al. (1995) and Boesgaard et al. (1998) who used HIRES at Keck to gather spectra of three stars in M 92; and Pasquini \& Molaro $(1996,1997)$ who exploited the presence of closer clusters in the southern emisphere to obtain spectra of a few stars in NGC 6397 and 47 Tuc using EMMI at NTT (these stars are about a magnitude brighter than those of M 92). However, very low $S / N$ observations of a handful of stars could be obtained. Only UVES (D'Odorico et al. 2000) at the Kueyen (VLT UT2) Telescope is able to provide abundances of $\mathrm{O}, \mathrm{Na}, \mathrm{Mg}, \mathrm{Al}$ and other elements for significant samples of well studied stars at the TO and at the base of RGB (well below the RGB bump), crucial to clarify most of these issues. Here we present a short description of the first results of the ESO Large Program 165.L-0263 devoted to the acquisition and analysis of high dispersion spectra ( $R \gtrsim 40000)$ of a fairly large sample of stars at the TO and at the base of the RGB in NGC 6752, NGC 6397, and 47 Tuc. A more detailed presentation of the analysis methods, and of related results for $\mathrm{Li}$, will be given in forthcoming papers, still in preparation.

\section{Sample selection and observations}

Observations were carried out in two 6-nights runs (June and September 2000); due to poor weather conditions in the September run, we were not able to complete the acquisition of the spectra in 47 Tuc. Hence the present paper deals only with data for the two other clusters. In the same runs we also acquired data for 25 field stars with good parallaxes from the Hipparcos catalogue and metallicity similar to that of the GCs.

For each cluster, we selected two groups of stars: (i) dwarfs about 0.2 mag brighter than the turn-off ( 5 stars in NGC 6397 and 9 in NGC 6752); and (ii) stars at the base of the red giant branch (3 stars in NGC 6397 and 9 stars in NGC 6752), hereinafter called subgiants. Positions of the observed stars in the $\mathrm{C}-\mathrm{M}$ diagrams of the two clusters (from Grundahl et al. 1999) are given in Fig. 1. A posteriori, radial velocities and chemical composition show that all stars are bona fide members of the clusters. Stars with likely strong and weak CN bands were selected from Strömgren photometry: the $c_{1}$ index seems in fact to be higher for CN-richer stars (see Grundahl et al. 1999). Given this choice, it should be clear that stars are not a random sample of stars in the different magnitude bins, but rather they were selected in order to include quite extreme cases of CN-poor and CN-rich stars. For comparison, we give in Fig. 2 also the location in a similar C-M diagram of the field stars we observed.

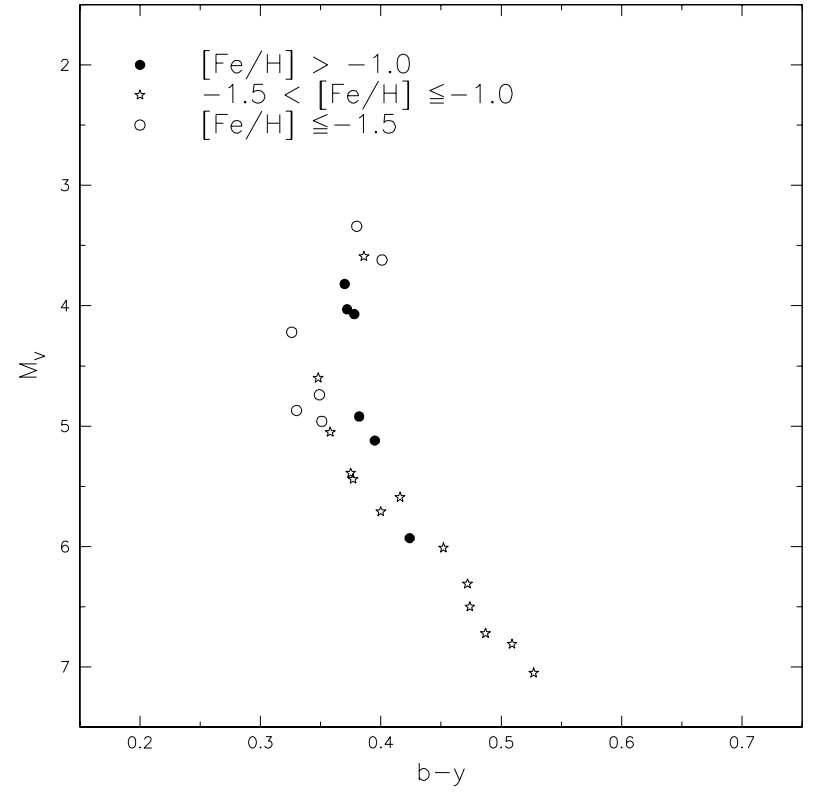

Fig. 2. Colour-magnitude diagram for the field stars; $M_{V}$ is computed using Hipparcos parallaxes

The observations were carried out using the dichroic beamsplitter \#2. This allowed us to observe simultaneously two spectral ranges with the two arms of the spectrograph. Slightly different setups were used in the two runs: the blue spectra cover the spectral range from about $3500 \AA$ to about $4700 \AA$, while the red spectra cover the range from $5700 \AA$ to about $8700 \AA$. This very broad spectral range allows observations of a large number of spectral features. The resolution was set by the slit width: in most cases this was 1 arcsec (yielding a resolution of 43000 at order centers); however, this value was sometimes changed according to the variable seeing conditions, from 0.7 arcsec in the best cases, down to $1.2 \operatorname{arcsec}$ in the worst ones. The slit length was always set at 8 arcsec, allowing accurate sky subtraction (necessary, since observations were carried out in full moon). Most of the objects are very faint $(V \gtrsim 17)$, so that long exposures were required even with an $8 \mathrm{~m}$ telescope like Kueyen, and a very efficient spectrograph like UVES. Typical exposure times were $\sim 1 \mathrm{hr}$ for subgiants, and about $4 \mathrm{hr}$ (split in 3 to 4 segments) for TO stars. The $S / N$ (per pixel at $6700 \AA$; there are about 5 pixels per resolution element) of the resulting spectra is $\sim 100$ in the best cases, and down to about 20 in the worst ones. Relevant data are presented in Tables $1-3$.

\section{Analysis}

Equivalent widths $(E W \mathrm{~s})$ for a large number of lines were measured using an authomatic procedure (similar to the one used in Bragaglia et al. 2001; full details will be given elsewhere). Typical errors in the $E W \mathrm{~s}$ (obtained by comparing values measured on different, similar stars) are $\pm 3 \mathrm{~m} \AA$ for NGC 6397 stars, and $\pm 5 \mathrm{~m} \AA$ for NGC 6752 stars, but they are as low as $\pm 2.5 \mathrm{~m} \AA$ in the best cases 
Table 1. Parameters for field stars

\begin{tabular}{rrrrrrrrr}
\hline Star & $M_{v}$ & $B-V$ & $b-y$ & {$[\mathrm{Fe} / \mathrm{H}]$} & $\log g$ & $\begin{array}{r}T_{\text {eff }} \\
\mathrm{H}_{\alpha}\end{array}$ & $\begin{array}{r}T_{\text {eff }} \\
\text { Best }\end{array}$ & $\begin{array}{r}T_{\text {eff }} \\
\text { AD/BD } / \text { B }\end{array}$ \\
\hline 10607 & 4.03 & 0.56 & 0.372 & -0.95 & 4.00 & 5780 & 5734 & \\
29907 & 6.01 & 0.63 & 0.452 & -1.47 & 4.82 & 5636 & 5406 & \\
31128 & 5.05 & 0.49 & 0.358 & -1.44 & 4.63 & 6279 & 5969 & \\
108177 & 4.87 & 0.43 & 0.330 & -1.70 & 4.44 & 5884 & 6063 & 6067 \\
116064 & 4.74 & 0.45 & 0.349 & -1.87 & 4.35 & 5770 & 5923 & \\
120559 & 5.93 & 0.66 & 0.424 & -0.90 & 4.51 & 5207 & 5378 & \\
121004 & 5.12 & 0.62 & 0.424 & -0.75 & 4.32 & 5401 & 5548 & \\
126681 & 5.71 & 0.60 & 0.400 & -1.13 & 4.55 & 5444 & 5556 & 5541 \\
132475 & 3.62 & 0.56 & 0.401 & -1.61 & 3.79 & 5596 & 5520 & 5788 \\
134169 & 3.82 & 0.565 & 0.370 & -0.81 & 4.06 & 6106 & 5825 & 5874 \\
134439 & 6.72 & 0.77 & 0.487 & -1.31 & 4.78 & 5151 & 5052 & 4974 \\
134440 & 7.05 & 0.85 & 0.527 & -1.28 & 4.69 & 4777 & 4777 & 4746 \\
140283 & 3.34 & 0.49 & 0.380 & -2.46 & 3.67 & 5560 & 5645 & 5691 \\
145417 & 6.81 & 0.82 & 0.509 & -1.23 & 4.78 & 5096 & 4922 & \\
159482 & 4.92 & 0.58 & 0.382 & -0.83 & 4.32 & 5546 & 5656 & \\
166913 & 4.22 & 0.45 & 0.326 & -1.59 & 4.08 & 5921 & 6020 & \\
181743 & 4.96 & 0.45 & 0.351 & -1.81 & 4.42 & 5749 & 5927 & \\
188510 & 5.59 & 0.60 & 0.416 & -1.44 & 4.50 & 5412 & 5485 & 5564 \\
189558 & 3.59 & 0.575 & 0.386 & -1.15 & 3.87 & 5829 & 5670 & 5663 \\
193901 & 5.44 & 0.55 & 0.377 & -1.03 & 4.62 & 5848 & 5780 & 5750 \\
194598 & 4.60 & 0.49 & 0.348 & -1.15 & 4.42 & 6064 & 5980 & 6017 \\
204155 & 4.07 & 0.57 & 0.378 & -0.70 & 4.06 & 5907 & 5751 & \\
205650 & 5.39 & 0.51 & 0.375 & -1.12 & 4.52 & 5628 & 5782 & \\
-350360 & 6.31 & 0.76 & 0.472 & -1.08 & 4.52 & 4957 & 5086 & 4980 \\
+053640 & 6.50 & 0.74 & 0.474 & -1.15 & 4.62 & 4987 & 5063 & \\
\hline
\end{tabular}

Table 2. Data for NGC 6397 stars (identifications from Grundahl et al. 1999); Al abundances from resonance lines

\begin{tabular}{|c|c|c|c|c|c|c|c|c|c|c|c|c|}
\hline Star & $S / N$ & $\begin{array}{r}n . \\
\text { lines }\end{array}$ & {$[\mathrm{Fe} / \mathrm{H}]$} & $\mathrm{rms}$ & $\begin{array}{l}E W \\
7771\end{array}$ & $\begin{array}{r}{[\mathrm{O} / \mathrm{Fe}]} \\
\mathrm{LTE}\end{array}$ & $\begin{array}{r}{[\mathrm{O} / \mathrm{Fe}]} \\
\mathrm{nLTE}\end{array}$ & $\begin{array}{r}E W \\
8183\end{array}$ & $\begin{array}{l}E W \\
8194\end{array}$ & $\begin{array}{r}{[\mathrm{Na} / \mathrm{Fe}]} \\
\mathrm{nLTE}\end{array}$ & {$[\mathrm{Mg} / \mathrm{Fe}]$} & $\begin{array}{r}{[\mathrm{Al} / \mathrm{Fe}]} \\
\mathrm{nLTE}\end{array}$ \\
\hline \multicolumn{13}{|c|}{ Dwarfs } \\
\hline 1543 & 91 & 23 & -2.02 & 0.12 & 14.7 & 0.32 & 0.16 & .. & 38.8 & 0.21 & 0.06 & 0.20 \\
\hline 1622 & 82 & 21 & -2.02 & 0.10 & 13.3 & 0.27 & 0.11 & 28.5 & 40.7 & 0.28 & 0.10 & 0.26 \\
\hline 1905 & 92 & 22 & -2.06 & 0.11 & 13.4 & 0.27 & 0.11 & 17.1 & 29.4 & 0.02 & 0.12 & 0.25 \\
\hline 201432 & 97 & 22 & -2.00 & 0.11 & 12.8 & 0.25 & 0.08 & 17.0 & 35.6 & 0.08 & 0.06 & 0.10 \\
\hline 202765 & 59 & 18 & -2.02 & 0.14 & 16.1 & 0.38 & 0.21 & 20.7 & 28.5 & 0.06 & -0.06 & 0.19 \\
\hline \multicolumn{13}{|c|}{ Subgiants } \\
\hline 669 & 91 & 27 & -2.01 & 0.11 & 6.0 & 0.34 & 0.26 & 62.9 & 86.8 & 0.48 & 0.23 & 0.46 \\
\hline 793 & 105 & 31 & -2.04 & 0.12 & $<6.0$ & $<0.34$ & $<0.26$ & 39.9 & 72.1 & 0.21 & 0.28 & 0.31 \\
\hline 206810 & 85 & 23 & -2.10 & 0.10 & 8.0 & 0.48 & 0.48 & 49.5 & 68.3 & 0.25 & 0.10 & 0.28 \\
\hline
\end{tabular}

(e.g. the Li lines at $6707 \AA$ ). As an example, Fig. 3 compares $E W$ s obtained for two TO-stars in NGC 6397 (stars 1543 and 1622) and two TO-stars in NGC 6752 (stars 4907 and 4428). From this comparison, we obtain rms of $3.4 \mathrm{~m} \AA$ and $4.8 \mathrm{~m} \AA$ respectively for typical $E W \mathrm{~S}$ (assuming errors are the same for the two stars). These values are comparable with results obtained for much brighter stars at other telescopes.

As discussed in Gratton et al. (1998), the single major source of error in the derivation of distances to globular clusters is the possible existence of systematic differences (of $0.015 \mathrm{mag}$ ) in the reddening scales for nearby subdwarfs and the much farther globular clusters. One of the aims of the present analysis was to derive accurate reddening estimates for GCs by comparing the observed colours with (reddening free) effective temperatures $T_{\text {eff's }}$ obtained from the spectra; for this reason our temperatures are based on fitting of the wings of the $\mathrm{H}_{\alpha}$ profiles with synthesized lines ${ }^{1}$ and not on colours. We wish to emphasize that the major concern here is the derivation of temperatures using a uniform procedure for both field and cluster stars; this is also important to ensure that the derived abundances may be directly comparable (again, a basic issue in the derivation of accurate distances to clusters). This approach also allows a proper discussion of

1 We only used $\mathrm{H}_{\alpha} . \mathrm{H}_{\beta}$ was not observed; the other members of the Balmer series were indeed observed, but they are more contamined by metal lines, and their dependence on gravity, metal abundance and details in the convection much larger than for $\mathrm{H}_{\alpha}$ (see Fuhrmann et al. 1993). 
Table 3. Data for NGC 6752 stars. Stars are ordered according to increasing Na abundance. Star identifications are from Grundahl et al. (1999). Note: 1) Al abundances from resonance lines; (2) Al abundances from high excitation 8772.9/73.9 doublet

\begin{tabular}{|c|c|c|c|c|c|c|c|c|c|c|c|c|}
\hline Star & $S / N$ & $\begin{array}{r}\mathrm{n} . \\
\text { lines }\end{array}$ & $\overline{[\mathrm{Fe} / \mathrm{H}]}$ & $\mathrm{rms}$ & $\begin{array}{c}E W \\
7771\end{array}$ & $\begin{array}{r}{[\mathrm{O} / \mathrm{Fe}]} \\
\text { LTE }\end{array}$ & $\begin{array}{r}{[\mathrm{O} / \mathrm{Fe}]} \\
\text { nLTE }\end{array}$ & $\begin{array}{c}E W \\
8183\end{array}$ & $\begin{array}{c}E W \\
8194\end{array}$ & $\begin{array}{r}{[\mathrm{Na} / \mathrm{Fe}]} \\
\mathrm{nLTE}\end{array}$ & {$[\mathrm{Mg} / \mathrm{Fe}]$} & $\begin{array}{r}{[\mathrm{Al} / \mathrm{Fe}]} \\
\mathrm{nLTE}\end{array}$ \\
\hline \multicolumn{13}{|c|}{ Dwarfs } \\
\hline 4428 & 49 & 22 & -1.52 & 0.14 & 35.7 & 0.35 & 0.24 & 24.4 & 57.1 & -0.35 & 0.04 & $-0.48(1)$ \\
\hline 4383 & 49 & 23 & -1.42 & 0.19 & 46.0 & 0.60 & 0.49 & 33.6 & 61.5 & -0.23 & 0.01 & $-0.11(1)$ \\
\hline 202316 & 44 & 21 & -1.56 & 0.20 & 28.7 & 0.31 & 0.20 & 42.6 & 69.0 & -0.09 & -0.06 & $-0.45(1)$ \\
\hline 4341 & 42 & 23 & -1.57 & 0.26 & 21.6 & 0.30 & 0.21 & 63.4 & 83.7 & 0.18 & 0.06 & $-0.76(1)$ \\
\hline 4458 & 33 & 17 & -1.52 & 0.27 & 19.4 & 0.04 & -0.06 & 71.9 & 82.6 & 0.24 & -0.09 & $-0.63(1)$ \\
\hline 4661 & 21 & 16 & -1.24 & 0.17 & .. & .. & .. & 75.4 & 84.3 & 0.28 & 0.15 & $-0.09(1)$ \\
\hline 5048 & 44 & 25 & -1.43 & 0.12 & 11.0 & -0.29 & -0.37 & 78.2 & 93.7 & 0.37 & -0.24 & 0.19 \\
\hline 4907 & 66 & 28 & -1.41 & 0.21 & 12.0 & -0.25 & -0.34 & 102.9 & 103.0 & 0.61 & -0.09 & $0.14(1)$ \\
\hline 200613 & 25 & 18 & -1.24 & 0.18 & .. & .. & .. & 103.5 & 110.1 & 0.64 & 0.06 & $0.60(1)$ \\
\hline \multicolumn{13}{|c|}{ Subgiants } \\
\hline 1406 & 48 & 44 & -1.39 & 0.16 & 18.0 & 0.40 & 0.34 & 82.1 & 105.7 & 0.02 & 0.20 & $0.10(2)$ \\
\hline 1665 & 49 & 41 & -1.34 & 0.15 & .. & .. & .. & 86.7 & 116.6 & 0.10 & 0.10 & $-0.13(2)$ \\
\hline 1445 & 35 & 33 & -1.53 & 0.13 & .. & .. & .. & 95.0 & .. & 0.19 & 0.06 & $0.33(2)$ \\
\hline 1563 & 44 & 38 & -1.42 & 0.17 & 17.4 & 0.38 & 0.33 & 97.0 & 131.0 & 0.25 & 0.11 & $0.17(2)$ \\
\hline 1400 & 70 & 41 & -1.44 & 0.14 & 15.3 & 0.33 & 0.28 & 103.4 & & 0.29 & 0.10 & $0.34(2)$ \\
\hline 1461 & 25 & 32 & -1.38 & 0.24 & .. & .. & .. & 94.9 & 144.4 & 0.29 & 0.10 & $-0.06(2)$ \\
\hline 202063 & 29 & 38 & -1.49 & 0.27 & .. & .. & .. & 120.4 & 143.8 & 0.44 & 0.13 & $0.33(2)$ \\
\hline 1481 & 55 & 46 & -1.39 & 0.15 & .. & .. & .. & 122.5 & 166.2 & 0.54 & -0.07 & $0.69(2)$ \\
\hline 1460 & 38 & 32 & -1.37 & 0.15 & $<14.0$ & $<0.25$ & $<0.19$ & 137.4 & .. & 0.65 & -0.19 & $0.86(2)$ \\
\hline
\end{tabular}

systematic differences between the chemical compostion of field and cluster stars. According to this approach, the systematic errors we will report are the possible offsets of groups of stars (e.g. those belonging to a cluster) with respect to the somewhat arbitrary scale defined by our field stars, used uniformly throughout our analysis.

The $\mathrm{H}_{\alpha}$ model lines were computed according to the precepts given in Castelli et al. (1997); throughout this paper we used model atmospheres extracted by interpolation within the grid by Kurucz with the overshooting option switched off (Kurucz 1993). Within these models, convection is considered using a mixing length approach, with a pressure scale height value of $l / H_{\mathrm{p}}=1.25$. It must be noted that it is not easy to derive accurate $\mathrm{H}_{\alpha}$ profiles from Echelle spectra, owing to the quite short free spectral range available for each order. We used the spectra extracted by the UVES pipeline; our procedure consisted in two steps:

1. we eliminated the high frequency variations due to the presence of contaminating lines by dividing the spectra for a contaminating line spectrum; this last was obtained by dividing the spectra for a spline traced through local fiducial continuum points obtained after an iterative clipping average over the highest half of the spectral points in small spectral intervals within the wings of $\mathrm{H}_{\alpha}$. To avoid possible errors due to the different $S / N$ of field and cluster stars, in this last case the contaminating line spectrum was obtained from the sum of all spectra for similar stars. Note that the $S / N$ (per pixel) of this summed spectra is $\geq 150$ that is similar to that obtained for the field stars;
2. we then compared the instrumental fluxes in regions at intermediate distances from the line center (typically 2 to $10 \AA$ ), with those obtained at large distances from the center (20 to $30 \AA$ ). These instrumental fluxes were then compared with those given by models for the same spectral regions. Note that the very central region of the line - the most critically affected by uncertainties in the model atmospheres - was not used in our comparisons. Also, our temperatures included appropriate corrections for the dependence of the profiles on gravity and metal abundance.

In the case of field stars, a comparison with temperatures obtained from colours (that have small internal errors) shows that errors of individual temperatures are $\sim 150$ $200 \mathrm{~K}$ for individual stars; nearly the same value is obtained by comparing values obtained for similar stars in globular clusters. These rather large errors are due to uncertainties in the flat fielding procedure, and are typical for such temperature derivations when only one Balmer line is used (see e.g. Fuhrmann et al. 1994).

Systematic errors in these temperatures might be not negligible, as shown by Barklem et al. (2000); however, as mentioned above, this is not our major concern, because, insofar temperatures are derived homogenously for cluster and field stars, such errors have very small impact on the present discussion, and on the derivation of distances and ages for globular clusters (discussed in a future paper). The adopted temperature scale is confirmed by analysis of line excitation: however, temperature from excitation have slightly larger error bars due to the limited range of excitation for lines measurable in warm, metal-poor dwarfs and subgiants. 

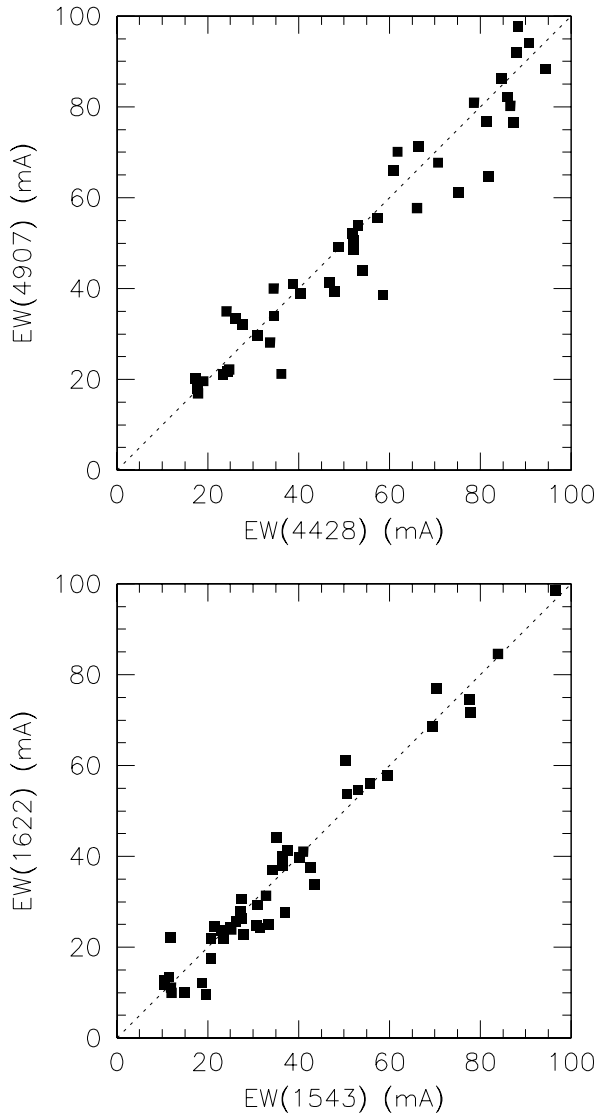

Fig. 3. Comparison between $E W$ s measured on the spectra of two dwarfs in NGC 6397 (stars 1543 and 1622), and two dwarfs in NGC 6752 (stars 4907 and 4428). The rms obtained from these comparisons are $3.4 \mathrm{~m} \AA$ for the stars in NGC 6397, and $4.8 \mathrm{~m} \AA$ for the stars in NGC 6752 (assuming equal error for both stars)

For field stars, we compared these $T_{\text {eff's }}$ (Col. 7 of Table 1) with those given by colours $(B-V$ and $b-y)$, using the calibration given by Kurucz (1993); here we assumed that all these nearby (field) stars are unreddened.

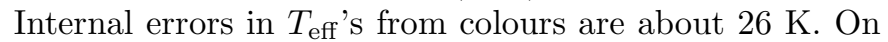
average, temperatures derived from $\mathrm{H}_{\alpha}$ are lower than those derived from colours by $97 \pm 38 \mathrm{~K}$, with a large r.m.s scatter of 188 K. For field stars, we then corrected the temperatures from colours to those derived from $\mathrm{H}_{\alpha}$, and averaged the two values, giving a weight 4 to temperatures from colours, and 1 to temperatures derived from $\mathrm{H}_{\alpha}$. These are our "best temperatures" for the field stars (Col. 8 of Table 1).

We may compare these "best temperatures" with those obtained by Alonso et al. (1996) using the IR flux method (Col. 9 of Table 1). Excluding the discrepant case of HD 132475 , on average our best $T_{\text {eff }}$ 's are lower than those given by Alonso et al. by $5 \pm 14 \mathrm{~K}$ (rms residuals $=45 \mathrm{~K}$, 10 stars). Hence our $T_{\text {eff }}$ 's can be assumed to be on the same scale of Alonso et al. Since temperatures from the IR flux method are rather robust with respect to possible errors in model atmospheres (although they are sensitive to uncertainties in the adopted reddening values), this
Table 4. Sensitivities of abundances to errors in the atmospheric parameters

\begin{tabular}{lccc}
\hline Element & $\begin{array}{r}\Delta T_{\text {eff }} \\
+100 \mathrm{~K}\end{array}$ & $\begin{array}{r}\Delta \log g \\
+0.2 \text { dex }\end{array}$ & $+0.2 \mathrm{~km} \mathrm{~s}$ s $^{-1}$ \\
\hline \multicolumn{3}{c}{ TO-star } \\
{$[\mathrm{Fe} / \mathrm{H}]$} & +0.096 & -0.054 & -0.050 \\
{$[\mathrm{O} / \mathrm{Fe}]$} & -0.161 & +0.123 & +0.048 \\
{$[\mathrm{Na} / \mathrm{Fe}]$} & -0.037 & +0.007 & +0.032 \\
{$[\mathrm{Mg} / \mathrm{Fe}]$} & -0.034 & +0.006 & +0.041 \\
{$[\mathrm{Al} / \mathrm{Fe}]$} & +0.001 & -0.012 & +0.007 \\
& \multicolumn{3}{c}{ Subgiants } \\
{$[\mathrm{Fe} / \mathrm{H}]$} & +0.108 & -0.023 & -0.049 \\
{$[\mathrm{O} / \mathrm{Fe}]$} & -0.200 & +0.098 & +0.044 \\
{$[\mathrm{Na} / \mathrm{Fe}]$} & -0.044 & -0.010 & +0.026 \\
{$[\mathrm{Mg} / \mathrm{Fe}]$} & -0.055 & +0.020 & +0.043 \\
{$[\mathrm{Al} / \mathrm{Fe}]$} & -0.080 & +0.021 & +0.038 \\
\hline
\end{tabular}

comparison shows that systematic errors in our temperature scale from $\mathrm{H}_{\alpha}$ profiles are likely not very large.

When plotted one on top of the other (separately: dwarfs and subgiants, and stars in different clusters), the $\mathrm{H}_{\alpha}$ profiles for cluster stars look undistinguishable. Furthermore, we were unable to find any correlation between colours and temperatures either from line excitation or $\mathrm{H}_{\alpha}$ profiles. We then concluded that the stars are intrinsically very similar, and that the slightly different values of colours and temperatures we derived for each star are due to random errors. In the following analysis, we have then adopted for all stars in these groups the same average temperatures (note however that this assumption is not critical in the present discussion).

Surface gravities $\log g$ were obtained from the location of the stars in the colour magnitude diagram, assuming masses consistent with an age of 14 Gyr (again, this assumption is not critical). As usual, microturbulent velocities were obtained by eliminating trends of abundances derived from individual Fe lines with expected line strength. Star-to-star scatter in Fe abundances within each group (same cluster, same evolutionary phase) were reduced by adopting for all stars the same microturbulent velocity; these average values were then adopted in the final analysis.

The finally adopted atmospheric parameters (effective temperatures in $\mathrm{K}$ /surface gravities/model metal abundances/microturbulent velocities in $\mathrm{km} \mathrm{s}^{-1}$ ) were as follows: NGC 6397 TO-stars (6476/4.10/-2.04/1.32); NGC 6397 subgiants $(5478 / 3.42 /-2.04 / 1.32)$; NGC 6752 TO-stars $(6226 / 4.28 /-1.43 / 0.70)$; NGC 6752 subgiants $(5347 / 3.54 /-1.43 / 1.10)$.

Sensitivities of abundances to errors in the atmospheric parameters are given in Table 4. Errors in final abundances are mostly due to possible errors in the adopted $T_{\text {eff }}$ 's. For our line list (dominated by low excitation lines), Fe I abundances rise by $0.10 \mathrm{dex},[\mathrm{O} / \mathrm{Fe}]$ values by $-0.20 \mathrm{dex}$, and $[\mathrm{Na} / \mathrm{Fe}]$ ones by -0.04 dex for a $100 \mathrm{~K}$ increase in the adopted $T_{\text {eff. }}$. Systematic errors in $T_{\text {eff's }}$ are dominated by uncertainties in the fitting of 
the $\mathrm{H}_{\alpha}$ profiles: they are about $\pm 90 \mathrm{~K}$ for the dwarfs and $\pm 60 \mathrm{~K}$ for the subgiants ${ }^{2}$, leading to errors in the $[\mathrm{Fe} / \mathrm{H}]$ values of \pm 0.09 dex and \pm 0.06 dex respectively for TO-stars and subgiants. This value, appropriate for cluster stars, is smaller than that given for field stars simply

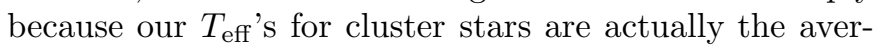
age over the values obtained for several stars, and errors for individual stars are given by uncertainties in the flat fielding procedure, that are only weakly affected by $S / N$. Corresponding errors in $[\mathrm{O} / \mathrm{Fe}]$ 's are \pm 0.18 and \pm 0.12 dex; those in $[\mathrm{Na} / \mathrm{Fe}]$ are \pm 0.04 and \pm 0.03 dex. However, in the context of the $\mathrm{O}-\mathrm{Na}$ anticorrelation, errors in temperatures adopted for individual stars are more important. In the case of NGC 6397, a quite realistic estimate can be obtained by the star-to-star scatter in Fe abundances, that is 0.032 dex, corresponding to an rms spread of $33 \mathrm{~K}$ in the $T_{\text {eff's. }}$ The adoption of a uniform temperature would then yield errors of $\pm 0.07 \mathrm{dex}$ in $[\mathrm{O} / \mathrm{Fe}]$ 's and $\pm 0.01 \mathrm{dex}$ in $[\mathrm{Na} / \mathrm{Fe}]$ 's (to be compared with the observed star-tostar scatter). In the case of NGC 6752, the star-to-star scatter of $0.096 \mathrm{dex}$ in $[\mathrm{Fe} / \mathrm{H}]$ 's also includes an important contribution due to noise in the $E W_{\mathrm{s}}$ : in fact the rms reduces to 0.074 dex if only spectra with $S / N>40$ are considered. We conclude that errors in individual $T_{\text {eff }}$ 's are $\lesssim 73 \mathrm{~K}$. Related errors in $[\mathrm{O} / \mathrm{Fe}]$ and $[\mathrm{Na} / \mathrm{Fe}]$ abundances are $\lesssim 0.15$ and $\lesssim 0.03$ dex. Again, these are the values to be compared with the observed scatter.

\section{Iron abundances}

Fe abundances were derived using a rather large number of lines, typically $\sim 20$ and $\sim 30-40$ for TO-stars and subgiants respectively. Full details about the line list, including $g f$ 's, $E W$ s and abundances from individual lines, will be given in separate papers (in preparation).

The Fe abundance obtained for dwarfs in NGC 6397 $([\mathrm{Fe} / \mathrm{H}]=-2.02 \pm 0.01$, where this error bar is the internal error as given by the standard deviation of the mean) is in good agreement with that determined from subgiants $([\mathrm{Fe} / \mathrm{H}]=-2.05 \pm 0.03)$. The average of the two groups is $[\mathrm{Fe} / \mathrm{H}]=-2.03 \pm 0.02$ (this is the internal error of our analysis, obtained by combining results for TOstars and subgiants; systematic errors, within the abundance scale defined by observations of the field stars, are mainly due to uncertainties in the adopted temperature; again combining results for TO stars and subgiants, that may be considered as independent each other, they are $\pm 0.04 \mathrm{dex})$. This value is less than that derived from giants

\footnotetext{
${ }^{2}$ The errors for $T_{\text {eff }}$ 's we quote for cluster stars are smaller than those we give for - individual - field stars, in spite of the lower $S / N$ values. This is because errors in individual $T_{\text {eff's }}$ 's are due to the flat fielding, and are then only marginally dependent on $S / N$; on the other side, temperatures for cluster stars are obtained averaging over the values obtained for all similar stars: for instance, in the case of NGC 6752 TO-stars, the adopted $T_{\text {eff }}$ is the average of the values obtained for nine individual stars. Star-to-star scatter in these $T_{\text {eff' }}$ is similar to the error bar we obtain for individual field star.
}

in Carretta \& Gratton (1997): $[\mathrm{Fe} / \mathrm{H}]=-1.82 \pm 0.04)$, and Zinn \& West (1984: $[\mathrm{Fe} / \mathrm{H}]=-1.91)$. However it agrees very well with the value obtained by Minniti et al. (1993: $[\mathrm{Fe} / \mathrm{H}]=-1.96 \pm 0.04)$, and the recent, comprehensive analysis of giants and subgiants by Castilho et al. (2000: $-2.00 \pm 0.05)$.

The equilibrium of ionization is not well reproduced: abundances from neutral Fe lines are 0.11 dex larger than those from singly ionized Fe lines. The same result is obtained for NGC 6752 and our field stars. Note that a smaller difference $(0.07 \mathrm{dex})$ in the same sense is also present in our solar reference analysis (in that case we obtained $\log n(\mathrm{Fe})=7.52$ from Fe I lines, and 7.45 from Fe II lines using the solar model atmosphere from Kurucz 1993, with no overshooting). We then think most of the difference for the Sun is due to either the adopted $g f$ 's values (these are laboratory values taken from recent literature) or to the model atmospheres (models might underestimate the temperature gradient in real atmospheres, perhaps due to an inappropriate consideration of convection). The residual difference for the program stars might be due to the adoption of slightly too high $T_{\text {eff }}$ 's $(\sim 40$ $50 \mathrm{~K}$ ) or too low gravities (by $\sim 0.1 \mathrm{dex}$ ). Note that it cannot be due to departures from LTE, because the expected dominating effect (overionization: Idiart \& Thévenin 1999; Gratton et al. 1999) would lead to larger abundances from Fe II lines than from Fe I ones (opposite to observations).

The star-to-star scatter in $[\mathrm{Fe} / \mathrm{H}]$ values is extremely small: the rms scatter is only 0.04 dex (i.e. 10\%) for NGC 6397. This seems a very homogeneous cluster as far as Fe abundances are considered.

On the other side, the $[\mathrm{Fe} / \mathrm{H}]$ value for NGC 6752 $([\mathrm{Fe} / \mathrm{H}]=-1.42 \pm 0.02$, internal error; systematic error is again \pm 0.04 dex), obtained both from TO and subgiant stars, which agree completely, coincides with that derived from giants by Carretta \& Gratton $(1997$ : $[\mathrm{Fe} / \mathrm{H}]=$ $-1.42 \pm 0.02)$; it is somewhat larger than the value quoted by Zinn \& West $([\mathrm{Fe} / \mathrm{H}]=-1.54)$ and Minniti et al. (1993: $[\mathrm{Fe} / \mathrm{H}]=-1.58 \pm 0.04)$. The spectra of NGC 6752 have a $S / N$ lower than those in NGC 6397 , since we chose to observe more stars, even at a lower $S / N$. The scatter of abundances for individual lines (from 0.12 to $0.27 \mathrm{dex}$ ) is larger than that obtained for stars in NGC 6397, roughly in agreement with the lower $S / N$.

For field stars we may compare the present Fe abundances with those derived by Carretta et al. (2000). Limiting ourselves to only those stars for which Carretta et al. considered high dispersion abundances, the present Fe abundances are smaller on average by $-0.05 \pm 0.02 \mathrm{dex}$ (11 stars, rms $=0.08 \mathrm{dex}$ ). The slightly lower metal abundances are due to lower $T_{\text {eff }}$ 's adopted in the present paper.

\section{The $\mathrm{O}-\mathrm{Na}$ and $\mathrm{Mg}-\mathrm{Al}$ anticorrelation}

In our analysis, oxygen abundances were derived from the permitted infrared triplet (7771-74 $\AA$ ): the forbidden lines are too weak in the program stars to be reliably measured, and we did not observe the region of the $\mathrm{OH}$ band in the 


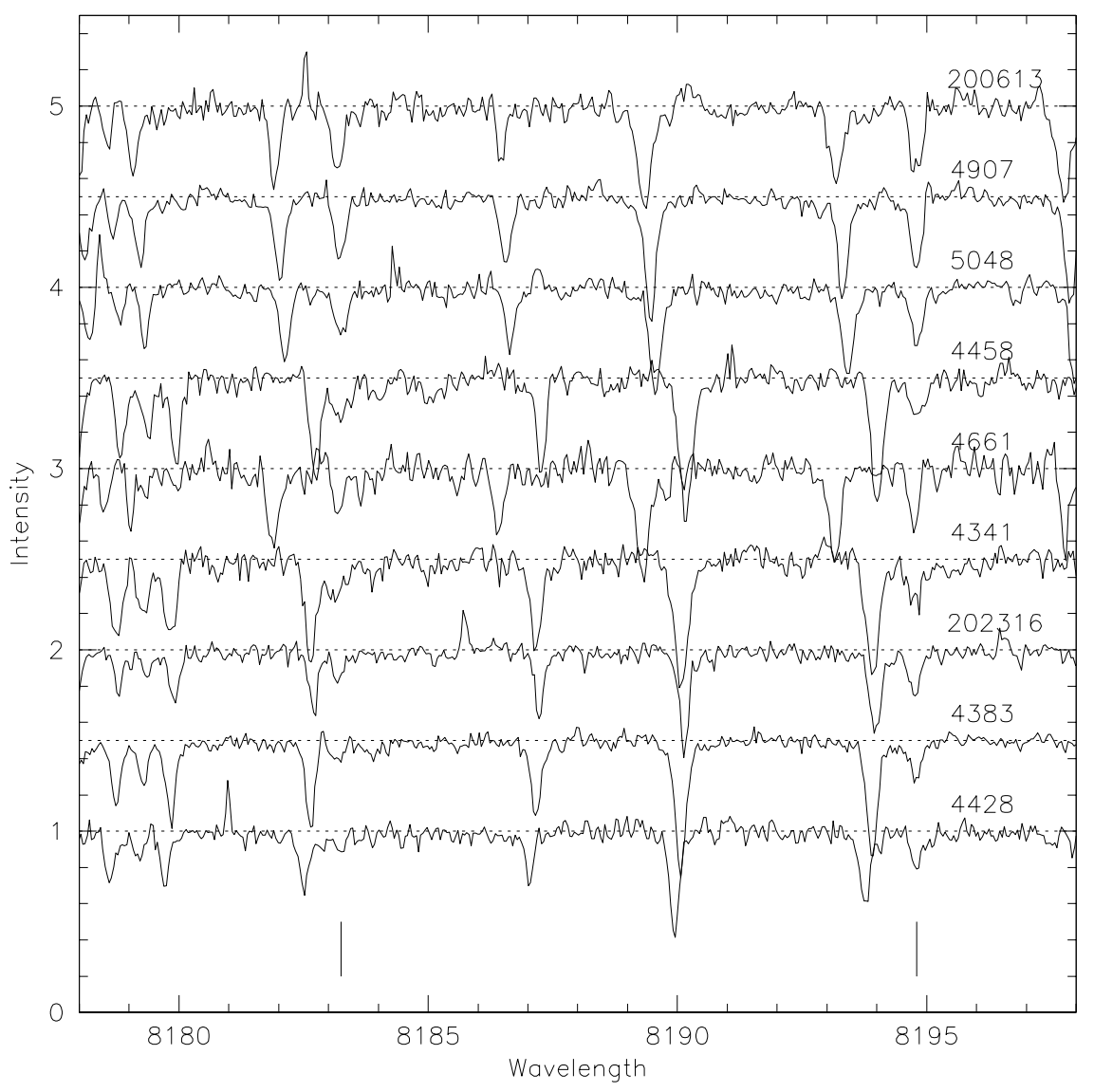

Fig. 4. Spectral region including the 8183-94 ^ Na I doublet in NGC 6752 TO-stars (stars are ordered according to decreasing $\mathrm{Na}$ abundances). The position of the Na lines is marked. Note that all these stars essentially have the same temperature, gravity, overall metal abundance and microturbulent velocity, so that observed variations in the line strengths can be directly interpreted as spread in the abundances

near UV because the required exposure times would be prohibitive even for an 8 meter telescope. Na abundances are based on the quite strong doublet at 8183-94 $\AA$, clearly visible in all stars. We checked that telluric lines were not blended with the stellar features. For both $\mathrm{O}$ and $\mathrm{Na}$, our abundances included non-LTE corrections, computed following the precepts of Gratton et al. (1999): however, these corrections are small and they do not affect any of the conclusion reached in this paper. $\mathrm{Mg}$ abundances are based on a few high excitation lines in the blue and yellow portion of the spectrum (typically three to four lines were measured for each star). Finally, whenever possible $\mathrm{Al}$ abundances were measured using the high excitation IR doublet at $8772.9 / 73.9 \AA$. However, this was possible only for subgiants in NGC 6752 , since in the other cases this doublet is too weak, and the only chance to measure $\mathrm{Al}$ abundances is by using the resonance doublet at 3944/61 A. These lines, while stronger, are not ideal abundance indicators due to saturation and the presence of large departures from LTE (see e.g. François 1986; Beyley $\&$ Cottrell 1987; and the discussion in Gratton \& Sneden 1988). An extensive discussion of departures from LTE for Al has been presented by Baumüller \& Gehren (1997): these authors found that rather large $(\sim 0.6 \mathrm{dex})$ non-LTE corrections are required for the resonance lines for model atmosphere parameters appropriate for the program stars, in the sense that an LTE analysis should grossly underestimate $\mathrm{Al}$ abundances. Application of such corrections greatly improve the agreement between abundances from the resonance lines and those derived from the 8772.9 $73.9 \AA$ doublet (for which non-LTE corrections are small). Such corrections were then included in the abundances listed in Tables 2 and 3. Although these large corrections might be somewhat uncertain, analysis of these lines may still be useful to discuss the $\mathrm{Mg}-\mathrm{Al}$ anticorrelation insofar only stars with very similar atmospheric parameters are considered, because in this case we expect that departures from LTE should be essentially the same for all such stars.

For NGC 6397, the $\mathrm{O}$ abundance is $[\mathrm{O} / \mathrm{Fe}]=+0.21 \pm$ 0.05 (internal error; systematic errors are $\sim 0.1 \mathrm{dex}$ ), and the average $[\mathrm{Na} / \mathrm{Fe}]$ ratio is $[\mathrm{Na} / \mathrm{Fe}]=+0.20 \pm 0.05$. This $\mathrm{O}$ excess is quite small in comparison to the values usually found for metal-poor stars (see e.g. Gratton et al. $2000)$, but it agrees very well with the mean values determined by Minniti et al. (1997): $[\mathrm{O} / \mathrm{Fe}]=+0.19)$ and Norris \& Da Costa (1995: [O/Fe] 0.1) from analysis of the forbidden lines in red giant spectra. Also the $\mathrm{Mg}$ abundance we get is quite low: this point will be discussed in a next paper. On the other side, the $[\mathrm{Na} / \mathrm{Fe}]$ value we found agrees well with that determined for a single red giant by 


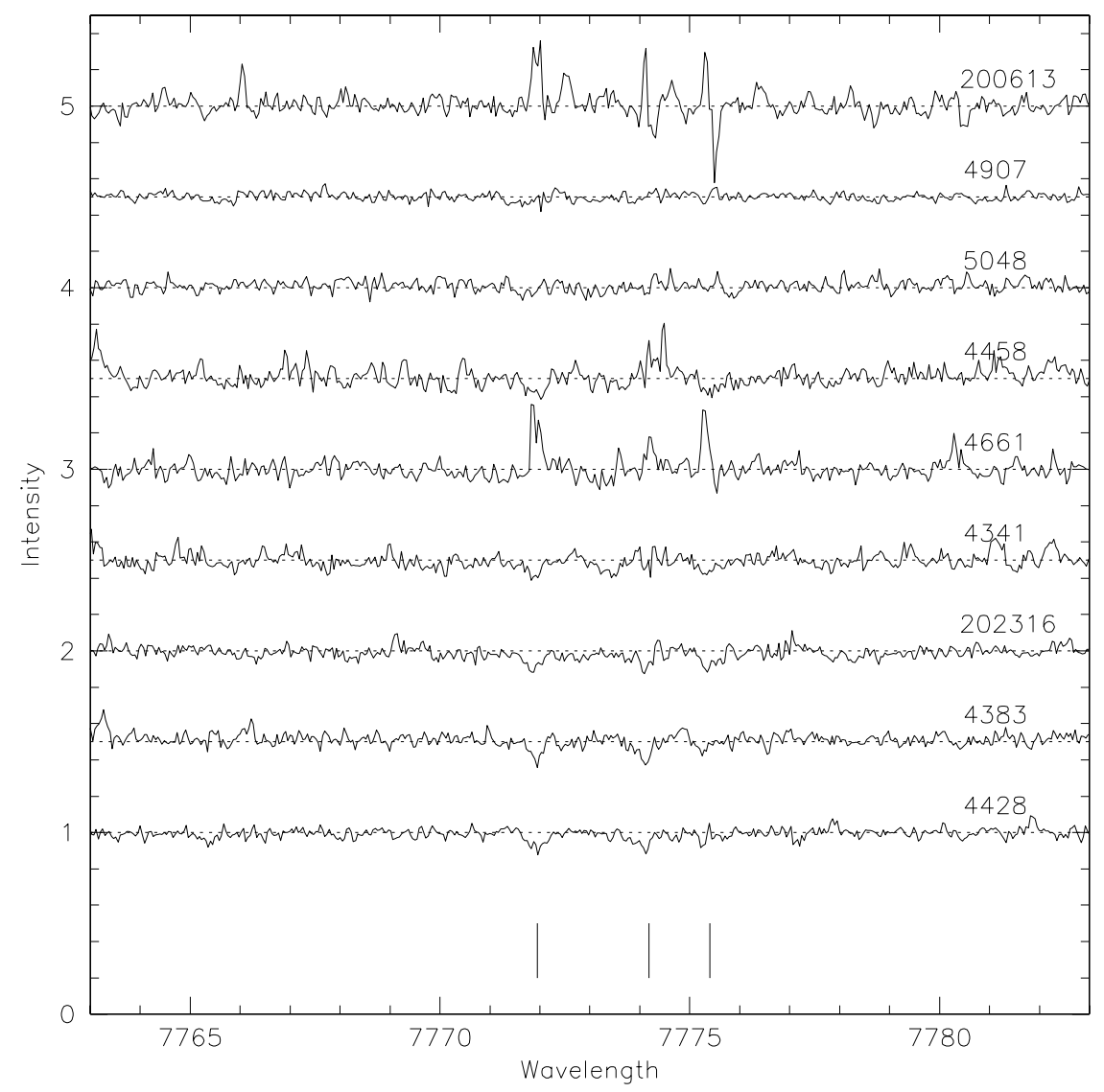

Fig. 5. Same as Fig. 3, but for the region including the 7771-74 A OI triplet in NGC 6752 TO-stars (stars are ordered according to decreasing $\mathrm{Na}$ abundances). The position of the $\mathrm{O}$ lines is marked

Carretta (1994: $[\mathrm{Na} / \mathrm{Fe}]=+0.22)$, and for two more by Norris \& Da Costa (1995: [Na/Fe] 0.2), while it is somewhat larger than the value of $[\mathrm{Na} / \mathrm{Fe}]=-0.01$ found by Minniti et al. (1997). We wish to remark that none of the program stars seem to be oxygen-poor and sodiumrich. The star-to-star scatter in our determinations $(0.15$ and 0.14 dex, rms, respectively) is larger than expected from errors in the $E W \mathrm{~s}$ alone (we would expect rms values of $\sim 0.10 \mathrm{dex}$ ): however it may be justified by some star-to-star scatter in the atmospheric parameters (within the measuring errors), not accounted for in our analysis (we are assuming that all dwarfs and subgiants may be analyzed using the same model atmospheres), and by a small offset in the results for dwarfs and subgiants (again, likely due to small errors in the adopted set of atmospheric parameters, within our quoted uncertainties). Given the small spread in Fe abundances, and the absence of a clear $\mathrm{O}-\mathrm{Na}$ anticorrelation, we conclude that NGC 6397 is indeed a very homogenous cluster. On the whole, our results indicate that stars in this cluster conforms to the paradigm set by field stars, confirming earlier findings by Bell \& Dickens (1980).

NGC 6752 presents a very different scenario (this is not too surprising, in view of the variations of the strength of $\mathrm{CN}$ and $\mathrm{CH}$ bands among stars on the MS already noticed by Suntzeff \& Smith 1991). There is a clear anticorrelation between $\mathrm{O}$ and $\mathrm{Na}$ abundances even for stars

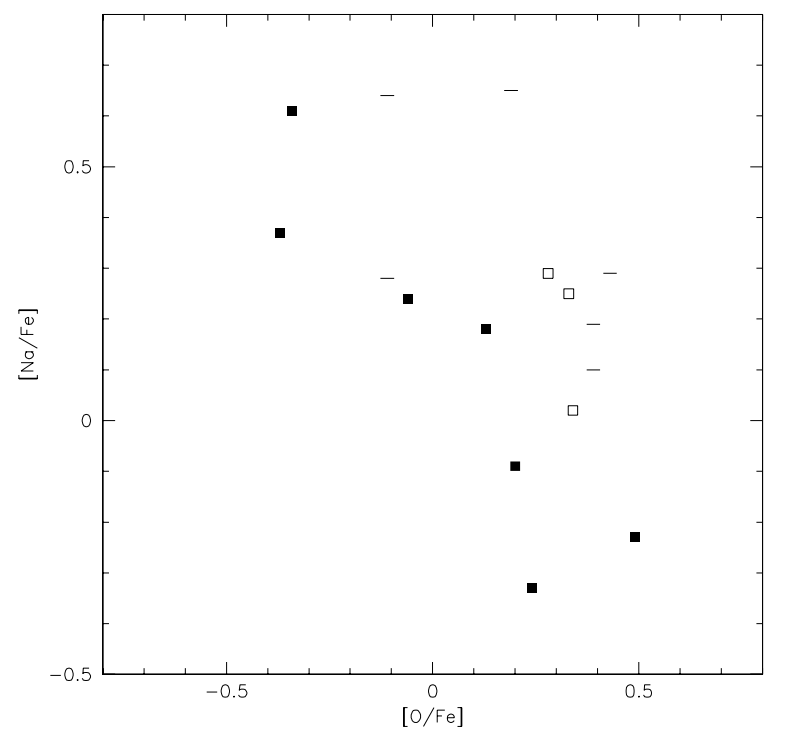

Fig. 6. Run of the abundances of $\mathrm{Na}$ and of $\mathrm{O}$ for stars in NGC 6752. Filled squares are TO-stars, open squares are subgiants, and lines represent upper limits (for $\mathrm{O}$ )

at the TO. This is clearly illustrated by Figs. 4 and 5, where we plotted the spectral regions including the $\mathrm{Na}$ and $\mathrm{O}$ lines in these stars (stars are ordered according to decreasing $\mathrm{Na}$ abundances). Notice that all these stars 


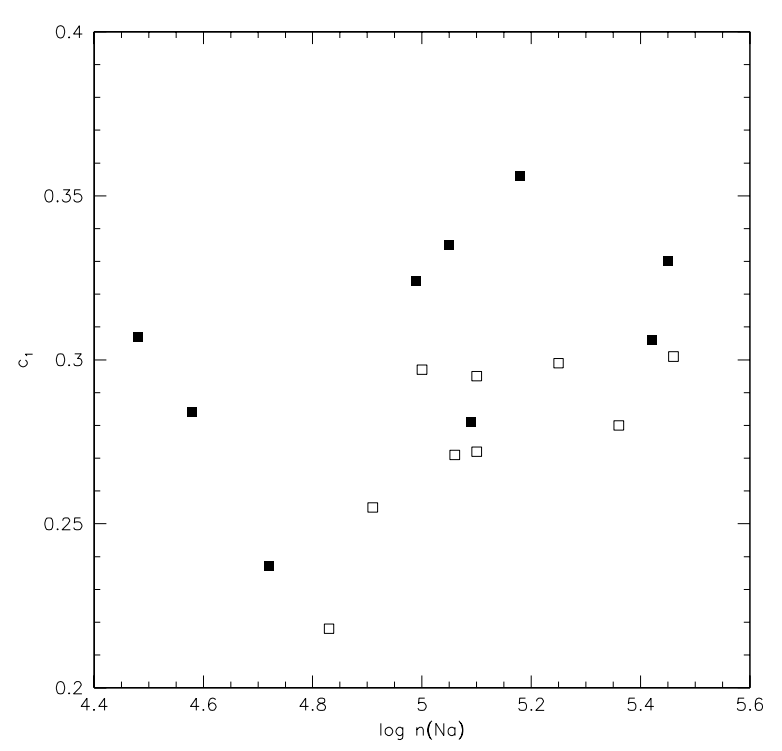

Fig. 7. Run of the $c_{1}$ index from Strömgren photometry with the Na abundance for stars in NGC 6752. Filled squares are TO-stars, and open squares are subgiants

have the same (or at least very similar) temperature, gravity, overall metal abundance and microturbulent velocity, so that observed variations in the line strengths may be directly interpreted as spread in the abundances: then, in spite of the fact that a few of the spectra are somewhat noisy, this result does not depend on details of the analysis, but rather is a solid purely observational evidence, largely independent of all the assumptions made. We found that $\mathrm{Na}$ abundances span a range of almost an order of magnitude. O abundances also change by a rather large factor; while not as extreme as in M 13, we found a rather extensive $\mathrm{O}-\mathrm{Na}$ anticorrelation in NGC 6752 too. This anti-correlation is shown in Fig. 6. There is a small offset in the O-Na anticorrelation between TO-stars and subgiants, but we think this is an artefact of the analysis (slightly incorrect offsets in temperatures and gravities).

There is some correlation between $c_{1}$ and the $\mathrm{O}-\mathrm{Na}$ trends (see Fig. 7). Note however that the scatter in this relation is quite large and the correlation is significant only for subgiants; this is not surprising, since our spectra shows that molecular bands are very weak in the spectra of TO-stars of NGC 6752, and their variations are not likely to affect significantly intermediate band colours like $c_{1}$.

Figure 8 displays results for $\mathrm{Mg}$ and $\mathrm{Al}$ in NGC 6752. The star-to-star variations in the $\mathrm{Al}$ abundances are large: $\sim 1$ dex among subgiants, and even more among TO-stars. These large variations cannot be explained by observational errors, and (for TO-stars) by any plausible differential non-LTE effect among stars with very similar atmospheric parameters. On the other side, star-tostar variations in the $\mathrm{Mg}$ abundances are much smaller ( $\lesssim 0.4 \mathrm{dex}$ ), and they may be seen clearly only among subgiants; for TO-stars, the used Mg lines are quite weak and a larger scatter in the abundances is due to errors in the $E W$ s. Data for subgiants, for which reliable $\mathrm{Al}$

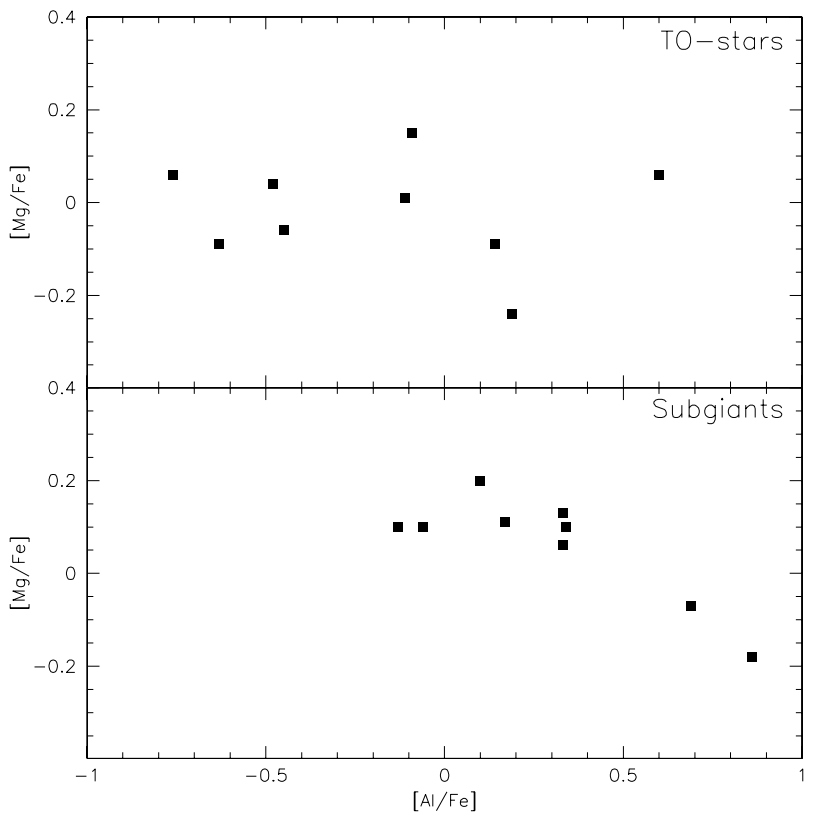

Fig. 8. Run of the abundances of $\mathrm{Mg}$ against that of $\mathrm{Al}$ for stars in NGC 6752. The upper panel shows results for TO-stars; the lower panel those for subgiants. Note that $\mathrm{Al}$ abundances for TO-stars were obtained using the resonance doublet, which is affected by large departures from LTE

abundances could be obtained from the high excitation $\mathrm{Al}$ I doublet at $8772.9 / 73.9 \AA$, indicate that also for these elements there is a clear anticorrelation between $\mathrm{Mg}$ and $\mathrm{Al}$. Stars 1460 and 1481 (the two most Na-rich subgiants) are clearly Mg-poor, Al-rich. We emphasize here that while the $\mathrm{Mg}-\mathrm{Al}$ anticorrelation is not obvious in panel a of Fig. 8, we think that such an anticorrelation exists also for TO-stars because the $\mathrm{Al}$ abundances show a very large star-to-star variation, correlated with $\mathrm{Na}$ abundance variations; we think the anticorrelation is less obvious in this case because we had to measure the resonance doublet of $\mathrm{Al}$ at 3944/61 $\AA$, not an ideal abundance indicator as we mentioned above, and moreover $\mathrm{Mg}$ abundances have larger errors, comparable to the star-to-star variations seen among subgiants.

We are now preparing line lists to study $\mathrm{CH}$ and $\mathrm{CN}$ abundances. However, it seems clear that the strength of these bands is correlated with $\mathrm{Na}$ and $\mathrm{O}$ abundances. Also, there is a correlation with the Stromgren $c_{1}$ index, albeit the scatter is quite large. Finally, in a forthcoming paper we will present a full discussion of the $\mathrm{Li}$ abundances in these stars. Here we anticipate that $\mathrm{Li}$ abundances in NGC 6752 stars seem also anticorrelated with the Na ones.

\section{Summary and conclusions}

We have presented the first results of the ESO Large Program 165.L-0263: we have used the UVES spectrograph on VLT2 to obtain high resolution $(R \gtrsim 40000)$ spectra for a quite large number of stars at the turn-off (14 stars between the two GCs) and the base of the giant 
branch (12 stars) in the globular clusters NGC 6397 and NGC 6752 . Thanks to the efficiency and wide spectral coverage of UVES we were able to obtain reliable $E W$ s for a number of lines of $\mathrm{Fe}, \mathrm{Li}, \mathrm{O}, \mathrm{Na}$, and other elements. The main results of this first analysis are:

(i) The $[\mathrm{Fe} / \mathrm{H}]$ value for $\mathrm{NGC} 6397$ is $[\mathrm{Fe} / \mathrm{H}]=-2.03 \pm$ $0.02 \pm 0.04$ (internal and systematic errors within the abundance scale defined by observations of the field stars); less than that derived from giants by Carretta \& Gratton (1997) and Zinn \& West (1984), but in agreement with the recent analysis of giants and subgiants by Castilho et al. (2000). The $[\mathrm{Fe} / \mathrm{H}]$ value for NGC $6752([\mathrm{Fe} / \mathrm{H}]=-1.42 \pm 0.02 \pm 0.04)$ coincides with the value derived from giants by Carretta \& Gratton (1997), and is slightly higher than the value quoted by Zinn \& West (1984);

(ii) In both clusters, $[\mathrm{Fe} / \mathrm{H}]$ obtained for TO stars agrees perfectly (within a few percent) with that obtained for stars at the base of the giant branch; this is a constraint on the impact of diffusion in stellar models. The starto-star scatter is extremely small: the rms scatter is only 0.04 dex (i.e. 10\%) for NGC 6397. This seems a very homogeneous cluster as far as Fe abundances are considered;

(iii) For NGC 6397, the $\mathrm{O}$ abundance is $[\mathrm{O} / \mathrm{Fe}]=+0.21 \pm$ 0.05 . This is a quite low value in comparison with those usually found in metal-poor stars, but it agrees well with previous determinations for the red giants of this cluster. The scatter of individual values is small, and none of the program stars seem to be oxygen-poor;

(iv) For NGC 6752, there is a clear anticorrelation between $\mathrm{Na}$ and $\mathrm{O}$ among $\mathrm{TO}$ stars and subgiants (similar to that seen among giants in this and other clusters). Also, an anticorrelation is observed between $\mathrm{Mg}$ and $\mathrm{Al}$, most clearly among subgiants, but likely existing also among TO-stars. Na-poor stars (i.e. stars with $[\mathrm{Na} / \mathrm{Fe}]<0.2)$ in NGC 6752 have $[\mathrm{O} / \mathrm{Fe}]=$ $+0.30 \pm 0.04$ ( 7 stars, rms $=0.10$ dex $)$. Extremes in $\mathrm{Al}$ abundances differ by over $1 \mathrm{dex}$, while for $\mathrm{Mg}$ the star-to-star scatter is smaller. The large variations in $\mathrm{Mg}$ and $\mathrm{Al}$ abundances suggests that nearly half of $\mathrm{Mg}$ has been converted into $\mathrm{Al}$ in the most Al-rich stars (1481 and 1460); note that these stars are also the most Na-rich ones. Note that given the adopted sample selection criteria, extreme cases of Na-poor and Na-rich stars may be overrepresented among observed stars.

We think the present results are very difficult to reconcile with deep mixing scenarios. To our knowledge, there are not appropriate calculations for main sequence stars. For giants, some calculations have been made by Langer et al. (1993) and Denissenkov \& Tout (2000): they show that the temperature required for p-capture on ${ }^{24} \mathrm{Mg}$ to finally produce the $\mathrm{Mg}$ - $\mathrm{Al}$ anticorrelation is $\sim 610^{7} \mathrm{~K}$; even if $\mathrm{Al}$ is produced starting from the far less abundant ${ }^{25} \mathrm{Mg}$ isotope, the temperature required is $\gtrsim 410^{7} \mathrm{~K}$; finally, the temperature required for extensive O-burning and Na production by p-capture on ${ }^{22} \mathrm{Ne}$ is $\sim 310^{7}$. All these values are much higher than expected even at the center of a globular cluster TO-star $\left(\begin{array}{llll}\sim 2 & 10^{7} & \mathrm{~K}\end{array}\right)$. Admittedly, these computations assume densities about 20 times lower than expected at the center of TO-stars, and much shorter timescales than MS lifetimes. However complete mixing of MS stars is unacceptable for several other reasons (e.g. it would bring large amount of fresh $\mathrm{H}$ to the center); furthermore, Li would be completely destroyed (while we see some Li even in O-depleted stars; paper in preparation). Hence, we think deep mixing scenarios cannot explain our results.

We are then forced to some primordial mechanism, like those proposed years ago by Cottrell \& Da Costa (1981) and D'Antona et al. (1983). In both scenarios, the inhomogeneities are due to the mass lost by intermediate mass stars $\left(M=4-5 M_{\odot}\right)$ during the Asymptotic Giant Branch (AGB) evolution and the planetary nebula expulsion: the two scenarios differ because Cottrell \& Da Costa think of a prolonged star formation, with most recently formed stars having a different chemical composition from the first ones; while D'Antona et al. consider pollution of the outer layers of already formed stars by other objects in the cluster. Anyhow, in both scenarios the intracluster gas is heavily nuclearly processed, due both to the occurrence of the third dredge-up from the helium buffer (Iben 1975) and by Hot Bottom Burning (HBB) at the basis of the convective envelopes of these massive AGB stars. Models for these evolutionary phases (Sackmann \& Boothroyd 1992; Ventura et al. 2000) successfully explain the occurrence and evolution of the lithium rich, oxygen rich massive AGBs in the Magellanic Clouds (Smith et al. 1995). Very recent models by Ventura et al. (2001) show that, in full stellar models computed for these intermediate mass stars at the low metallicities of Globular Clusters, the HBB temperature can reach values as large as $10^{8} \mathrm{~K}$. At this temperature, the complete $\mathrm{CNO}$ cycle operates, depleting oxygen. At the same time, p-captures on ${ }^{24} \mathrm{Mg}$ and ${ }^{20} \mathrm{Ne}$ produce $\mathrm{Al}$ (see also Denissenkov et al. 1998) and $\mathrm{Na}$. This model can then explain the anticorrelations O$\mathrm{Na}$ and $\mathrm{Mg}-\mathrm{Al}$. In this context it is very interesting to note that no O-Na anticorrelation is seen in NGC 6397. Also, $\mathrm{Li}$ abundances in this cluster follows the paradigma set by field metal-poor stars (Castilho et al. 2000). NGC 6397 is a quite small cluster (mass $\lesssim 10^{5} M_{\odot}$, from the integrated magnitude $M_{V}=-6.58$, Harris 1996, and a mass-to-light ratio of $\sim 2$, typical for a globular cluster). On the other side, the $\mathrm{O}-\mathrm{Na}$ anticorrelation is seen in the more massive cluster NGC 6752 (mass $\sim 210^{5} M_{\odot}$, from the integrated magnitude $M_{V}=-7.68$, Harris 1996, and the same mass-to-light ratio used for NGC 6397). A (cluster) mass threshold should be present in accretion scenarios (see e.g. Gratton 2001), and likely also in the prolonged star formation models. We plan to address thoroughly such problems in forthcoming papers.

Acknowledgements. This research has made use of the SIMBAD data base, operated at CDS, Strasbourg, France. We wish to thank V. Hill for help during the observations, and P. Bertelli for useful comments. We thank our referee 
(J. Cohen) for having provided very useful suggestions and data in advance of publication

\section{References}

Alonso, A., Arribas, S., \& Martinez-Roger, C. 1996, A\&A, 313, 873

Barklem, P. S., Piskunov, N., \& O’Mara, B. J. 2000, A\&A, 363, 1091

Baumüller, D., \& Gehren, T. 1997, A\&A, 325, 1088

Bell, R. A., \& Dickens, R. J. 1980, ApJ, 242, 657

Beyley, M. J., \& Cottrell, P. L. 1987, MNRAS, 224, 633

Boesgaard, A. M., Deliyannis, C. P., Stephens, A., \& King, J. R. 1998, ApJ, 493, 206

Bragaglia, A., Carretta, E., Gratton, R. G., et al. 2001, AJ, in press

Cannon, R. D., Croke, B. F. W., Bell, R. A., Hesser, J. E., \& Stathakis, R. A. 1998, MNRAS, 298, 601

Carretta, E. 1994, Ph.D. Thesis, Un. Padua

Carretta, E., \& Gratton, R. G. 1997, A\&AS, 121, 95

Carretta, E., Gratton, R. G., \& Sneden, C. 2000, A\&A, 356, 238

Castelli, F., Gratton, R. G., \& Kurucz, R. L. 1997, A\&A, 318, 841

Castilho, B. V., Pasquini, L., Allen, D. M., Barbuy, B., \& Molaro, P., 2000, A\&A, 361, 92

Cavallo, R. M., Sweigart, A. V., \& Bell, R. A. 1996, ApJ, 464, L79

Charbonnel, C. 1994, A\&A, 282, 811

Cohen, J. G. 1999a, AJ, 117, 2428

Cohen, J. G. 1999b, AJ, 117, 2434

Cottrell, P. L., \& Da Costa, G. S. 1981, ApJL, 245, L79

D'Antona, F., Gratton, R. G., \& Chieffi, A. 1983, MSAIt, 54, 173

Deliyannis, C. P., Boesgaard, A. M., \& King, J. R. 1995, ApJ, 452,12

Denissenkov, P. A., \& Denissenkova, S. N. 1990, SVAL, 16, 275

Denissenkov, P. A., \& Tout, C. A. 2000, MNRAS, 316, 395

Denissenkov, P. A., Da Costa, G. S., Norris, J. E., \& Weiss, A. 1998, A\&A, 333, 926

D'Odorico, S., Cristiani, S., Dekker, H., et al. 2000, in Discoveries and Research Prospects from 8- to 10-MeterClass Telescopes, ed. J. Bergeron, SPIE, 4005, 121

François, P. 1986, A\&A, 160, 264

Fuhrmann, K., Axer, M., \& Gehren, T. 1993, A\&A, 271, 451

Fuhrmann, K., Axer, M., \& Gehren, T. 1994, A\&A, 285, 585
Gratton, R. G. 2001, in The Chemical Evolution of the Galaxy, ed. F. Matteucci, \& F. Giovannelli (Kluwer, Dordrecht), in press

Gratton, R. G., \& Sneden, C. 1988, A\&A, 204, 193

Gratton, R. G., Carretta, E., \& Clementini, G. 1998, in PostHipparcos Cosmic Candles, ed. A. Heck \& F. Caputo (Kluwer, Dordrecht), 89

Gratton, R. G., Carretta, E., Eriksson, K., \& Gustafsson, B. 1999, A\&A, 350, 955

Gratton, R. G., Sneden, C., Carretta, E., \& Bragaglia, A. 2000, A\&A, 354, 169

Grundahl, F., Vandenberg, D. A., Stetson, P. B., Anderson, M. I., \& Briley, M. 1999, in The Galactic Halo: from Globular Clusters to Field Stars [astro-ph/9909447]

Harris, W. E. 1996, AJ, 112, 1487

Idiart, T., \& Thévenin, F. 1999, ApJ, 541, 207

Iben, I. Jr. 1975, ApJ, 196, 525

King, J., Stephens, A., \& Boesgaard, A. M. 1998, AJ, 115, 666

Kraft, R. P. 1994, PASP, 106, 553

Kraft, R. P., Sneden, C., Smith, G. H., et al. 1997, AJ, 113, 279

Kurucz, R. L. 1995, CD-ROM 13, Smithsonian Astrophysical Observatory, Cambridge

Langer, G. E., \& Hoffman, R. 1995, PASP, 107, 1177

Langer, G. E., Hoffman, R., \& Sneden, C. 1993, PASP, 105, 301

Minniti, D., Geisler, D., Peterson, R. C., \& Claría, J. J. 1993, ApJ, 413, 548

Minniti, D., Peterson, R. C., Geisler, D., \& Claría, J. J. 1996, ApJ, 470, 953

Norris, J. E. 1987, ApJ, 313, 65

Norris, J. E., \& Da Costa, G. S. 1995, ApJL, 441, L81

Osborn, W. 1971, Observatory, 91, 223

Pasquini, L., \& Molaro, P. 1996, A\&A, 307, 761

Pasquini, L., \& Molaro, P. 1997, A\&A, 322, 109

Sackmann, I. J., \& Boothroyd, A. I. 1992, ApJ, 392, L71

Smith, G. H. 1987, PASP, 99, 67

Smith, V. V., Plez, B., \& Lambert, D. L. 1995, ApJ, 441, 735

Suntzeff, N. B., 1981, ApJS, 47, 1

Suntzeff, N. B., \& Smith, V. 1991, ApJ, 381, 160

Sweigart, A. V., \& Mengel, J. G. 1979, ApJ, 229, 624

Ventura, P., D'Antona, F., \& Mazzitelli, I. 2000, A\&A, 363, 605

Ventura, P., D’Antona, F., Mazzitelli, I., \& Gratton, R. 2001, in preparation

Zinn, R., \& West, M. J. 1984, ApJS, 55, 45 